\title{
Papillary thyroid carcinoma presenting with cutaneous fungating mass complicated with myiasis: a case report
}

\author{
Ningombam Jiten Singh ${ }^{1}$, Sudhiranjan $\mathbf{T H}^{1}$, Lailyang $\mathbf{T}^{1}$, Anita $\mathbf{N}^{2}$ \\ ${ }^{1}$ Department of Otorhinolaryngology, Regional Institute of Medical Sciences, Imphal, Manipur, India \\ ${ }^{2}$ Department of Anaesthesiology, Regional Institute of Medical Sciences, Imphal, Manipur, India
}

Received: 28 July 2015

Accepted: 02 October 2015

\section{*Correspondence:}

Dr. Ningombam Jiten Singh,

E-mail: jiten.ningombam@gmail.com

Copyright: (c) the author(s), publisher and licensee Medip Academy. This is an open-access article distributed under the terms of the Creative Commons Attribution Non-Commercial License, which permits unrestricted non-commercial use, distribution, and reproduction in any medium, provided the original work is properly cited.

\begin{abstract}
Papillary Thyroid Carcinoma (PTC) is the most common type of thyroid malignancy making upto 70-80\% of all thyroid malignancies. PTC presenting with extrathyroidal cutaneous fungating mass is unusual. We report the management of a neglected fungating PTC which was complicated with myiasis. Such a case has not been reported earlier in the English literature. Preoperatively myiasis was managed conservatively following which she underwent total thyroidectomy with en-bloc resection of the fungating mass along with adequate skin margin and ipsilateral selective neck dissection. Postoperative period was uneventful and the patient was given radioiodine ablation therapy 4 weeks later.
\end{abstract}

Keywords: Papillary carcinoma thyroid, Extrathyroidal, Cutaneous fungating mass, Myiasis

\section{INTRODUCTION}

Papillary Thyroid Carcinoma (PTC) is the most common thyroid malignancy. PTC is often multifocal and metastasizes to regional lymph nodes in about $40 \%$ of cases. Distant metastasis of PTC is rare and usually involves the lungs, liver, bones, and brain. Cutaneous metastases occur in $0.6-10.4 \%$ of all patients with cancer and represent $2 \%$ of all skin tumours. ${ }^{1}$ Papillary thyroid carcinoma is a slow growing neoplasm which explains the relatively long duration until a diagnosis is established. Appropriate early surgical treatment decreases the risk of metastasis and recurrence. We report the management of a very rare case of neglected papillary carcinoma thyroid presenting with extrathyroidal cutaneous fungating mass infected with maggots.

\section{CASE REPORT}

A 58-year-old woman was referred with a history of a thyroid swelling for more than 20 years. She had papillary thyroid carcinoma diagnosed 3 years ago, for which a right hemithyroidectomy was attempted at a private hospital. However, the surgery was abandoned mainly due to technical inadequacy, and there was no follow-up. There was progression of the tumour on the right anterior neck that eventually developed as a fungating mass 6 months back. The mass was very foulsmelling and infested with maggots, for which she consulted an ENT surgeon, who referred the case to us.

On clinical examination, there was a large fungating mass measuring approximately $6 \mathrm{~cm} \times 5 \mathrm{~cm}$ on the right side. The right mass was infested with maggots and associated with foul-smelling, bloody discharge, thus causing great discomfort to the patient and family (Figure 1). Contrast enhanced Computed Tomography (CT) neck revealed a large and extensive thyroid mass with enlarged lymph nodes in the right cervical region. The carotid arteries, internal jugular veins, trachea and sternum appeared free (Figure 2). Preoperative vocal cord assessment revealed no obvious palsy of the vocal cords. Routine tests results 
including thyroid profile were within normal. Initial FNAC from the fungating mass showed organised haematoma. A repeat FNAC from the mass showed papillary carcinoma thyroid. Intravenous antibiotic cover was given preoperatively. Maggots were removed after application of turpentine oil locally. Preoperative haemoglobin was $6.6 \mathrm{~g} / \mathrm{dL}$, so two units of compatible blood were transfused before surgery. One more unit was transfused intra-operatively. An elliptical neck incision was made, taking the entire fungating mass together with a good healthy skin margin. Intraoperative findings revealed that the tumour had infiltrated the right strap and sternocleidomastoid muscles. The tumour mass was adhered to the right internal jugular vein and common carotid. The left lobe was removed first, preserving the left recurrent laryngeal nerve and parathyroid glands. The right fungating mass was then removed (Figure 3), along with right level II, III, IV and level V cervical nodes and right sternocleidomastoid and strap muscles and an extensive area of skin, leaving the trachea, IJV, common carotid artery and the right recurrent laryngeal nerve intact. Level VI nodes were also removed. The skin cover over the defect in lower neck was maintained after mobilising the local skin flap (Figure 4). The histopathology report confirmed the diagnosis of papillary thyroid carcinoma (Figure 5). Postoperative vocal cord assessment revealed no obvious palsy of the vocal cords. Postoperative serum calcium level was within normal. The patient underwent radioiodine ablation therapy 4 weeks later.

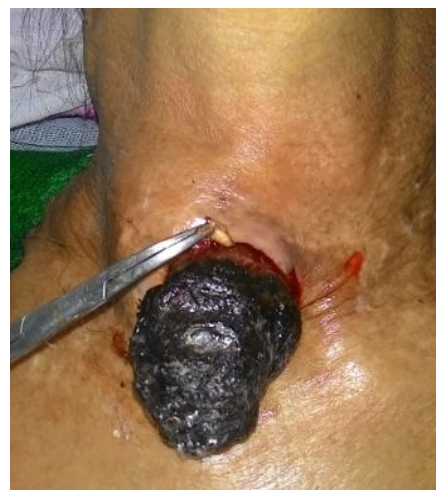

Figure 1: Preoperative extrathyroidal fungating mass with maggot infestation.

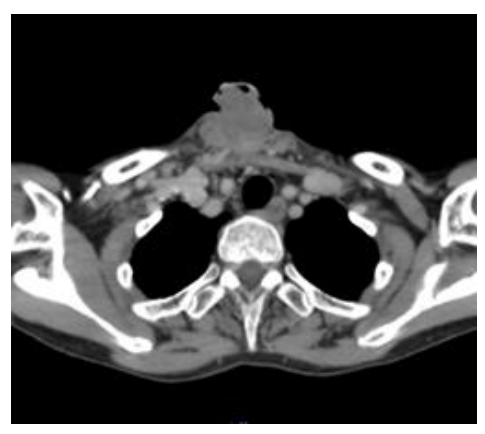

Figure 2: CT axial view showing tumour mass.

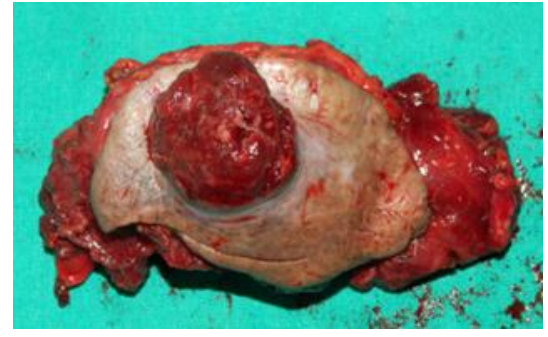

Figure 3: Showing the resected tumour mass.

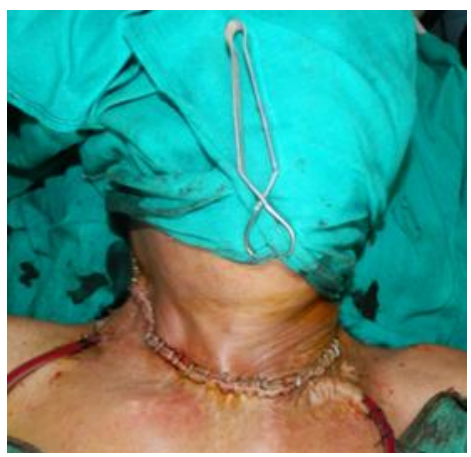

Figure 4: Post-operative after wound closure.

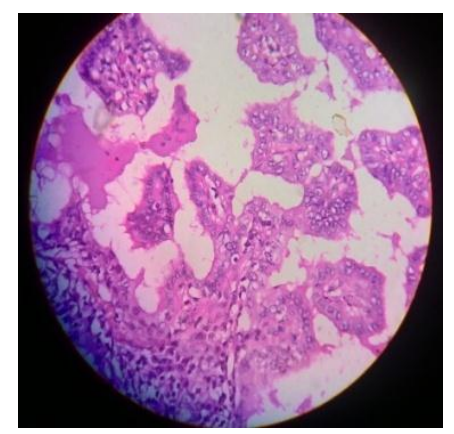

Figure 5: HPE showing papillary pattern and presence of intranuclear cytoplasmic inclusion.

\section{DISCUSSION}

PTC is the most common malignancy of thyroid gland. Well-differentiated PTC has an excellent prognosis, with a 5-year survival rate of greater than $97 \% .^{2}$ Extrathyroidal extension is seen in 4 to 16 percent of cases and carries with it an increased risk of disease recurrence and reduces overall survival rate. ${ }^{3}$ Extrathyroidal extension involves invasion of trachea, oesophagus, recurrent laryngeal nerve, strap muscles and skin. Cutaneous metastasis from thyroid carcinoma is rare. It usually occurs in the setting of disseminated neoplastic disease. ${ }^{4}$ Dahl et al reviewed the English literature from 1964 onwards and found 43 cases of thyroid carcinoma with skin metastases. They found that papillary carcinoma was the most common thyroid cancer to result in skin metastases, representing $41 \%$ of cases, followed by follicular carcinoma at $28 \%$, with anaplastic carcinoma and medullary carcinoma each contributing $15 \%$ of cases. The scalp was the most common site of involvement. ${ }^{4}$ 
Thyroid malignancy presenting with maggot infested cutaneous extrathyroidal mass is unusual. Such cases have been reported in anaplastic and follicular thyroid carcinoma. Nabawi AS et al has reported 11 cases with thyroid malignancy with cutaneous fungating lesion in which the final diagnosis showed DTC $=3$, poorly differentiated thyroid carcinoma $=5$, anaplastic cancer $=2$, medullary thyroid cancer $=5 .^{5}$ Our case report is a papillary thyroid carcinoma presented as maggot infested fungating extrathyroidal mass, and similar case has not been reported before in the English literature. Preoperative evaluation in our case required management of myiasis by local application of turpentine oil and antibiotic coverage for one week. Only after the clearance of the maggots and debri around the lesion the case was taken up for surgery.

Large fungating thyroidal masses are extremely rare and present a unique challenge to surgeons. These large masses often present in close proximity to or may involve a number of critical anatomical structures. Hence, before contemplating surgery, it is essential to perform a thorough work-up and assessment of the relevant anatomy and pathology. Most important in the preoperative diagnostic evaluation is to exclude anaplastic thyroid cancer, which has a dismal outcome and prognosis. PTC has favourable outcome even with cutaneous involvement.

A total thyroidectomy along with complete resection of the cutaneous mass and removal of the involved structures including cervical lymph nodes, followed by radioiodine ablation is the accepted management of PTC with extrathyroidal extension. Fujimoto $\mathrm{Y}$ et al. performed aggressive radical resection on 18 of 21 elderly patients with locally invasive-papillary carcinoma of the thyroid and advocated aggressive resection for control of locally advanced, differentiated carcinoma of the thyroid. ${ }^{6}$ Skin loss can either be closed primarily as in our case, or may require a flap. ${ }^{7}$ In the experienced hands, permanent complications such as recurrent laryngeal nerve injury and hypoparathyroidism occurs at a rate of less than $2 \% .^{8}$

In summary, managing a complicated Papillary Carcinoma Thyroid requires a thorough preoperative evaluation and work-up of the case. Any local infection like abscess or in rare cases like myiasis should be managed with regular wound debridement and proper antibiotic coverage. Surgery should address the primary disease, cutaneous spread, the nodal involvement and the involvement of the adjacent extrathyroidal structures. Post-operative radio-iodine ablation and suppressive doses of thyroxine and regular follow-up are also essential.

\section{ACKNOWLEDGMENTS}

The authors would like to thank all the faculty members of Otorhinolaryngology, Pathology and Radiology departments of Regional Institute of Medical sciences, Imphal, for their support in reporting this observation.

Funding: No funding sources

Conflict of interest: None declared

Ethical approval: Not required

\section{REFERENCES}

1. Alcaraz I, Cerroni L, Rütten A, Kutzner H, Requena L. Cutaneous metastases from internal malignancies: a clinicopathologic and immunohistochemical review. Am J Dermatopathol. 2012;34:347-93.

2. Howlader N, Noone AM, Krapcho M, Neyman N, Aminou R, Waldron W, et al. SEER cancer statistics review, 1975-2009 (Vintage 2009 Populations). Bethesda, MD: National Cancer Institute; 2012.

3. Andersen PE, Kinsella J, Loree TR, Shaha AR, Shah JP. Differentiated carcinoma of the thyroid with extrathyroidal extension. Am J Surg. 1995;170:467-70.

4. Dahl PR, Brodland DG, Goellner JR, Hay ID. Thyroid carcinoma metastatic to the skin: a cutaneous manifestation of a widely disseminated malignancy. J Am Acad Dermatol. 1997;36:531-7.

5. Nabawi AS, Al Wagih HF, Hemeida MA, Koraitim TY, Moussa M, Ezzat T. Fungating thyroid cancer: a complex clinical scenario. World J Surg. 2012 Mar;36(3):598-606.

6. Fujimoto Y, Obara T, Ito Y, Kodama T, Yashiro T, Yamashita T, et al. Aggressive surgical approach for locally invasive papillary carcinoma of the thyroid in patients over forty-five years of age. Surgery. 1986 Dec;100(6):1098-107.

7. Nakahira M, Nakatani H, Takeuchi S, Higashiyama, K, Fukushima K. Safe reconstruction of a large cervico-mediastinal tracheal defect with a pectoralis major myocutaneous flap and free costal cartilage grafts. Auris Nasus Larynx. 2006;33:203-6.

8. Mazeh H, Chen H. Advances in surgical therapy for thyroid cancer. Nat Rev Endocrinol. 2011;7:581-8.

Cite this article as: Jiten Singh N, Sudhiranjan TH, Lailyang T, Anita N. Papillary thyroid carcinoma presenting with cutaneous fungating mass complicated with myiasis: a case report. Int J Otorhinolaryngol Head Neck Surg 2015;1:85-7. 